\title{
Erratum to: Behavior change interventions and policies influencing primary healthcare professionals' practice-an overview of reviews
}

Bhupendrasinh F. Chauhan ${ }^{1,2,3^{*}}$, Maya M. Jeyaraman ${ }^{3}$, Amrinder Singh Mann ${ }^{3}$, Justin Lys ${ }^{3}$, Becky Skidmore ${ }^{4}$, Kathryn M. Sibley ${ }^{3,5}$, Ahmed M. Abou-Setta ${ }^{3,5}$ and Ryan Zarychanski ${ }^{3,5,6,7}$

\section{Erratum}

After publication of the original article [1] it was brought to our attention that authors Maya M. Jeyaraman, Ahmed M. Abou-Setta and Ryan Zarychanski were incorrectly included as Maya Jeyaraman, Ahmed Abou-Setta and Ryan Zarychanksi. The corrected names are included in the author list of this erratum.

\begin{abstract}
Author details
'College of Pharmacy, University of Manitoba, Winnipeg, Canada. ${ }^{2}$ Children's Hospital Research Institute of Manitoba, Winnipeg, Canada. ${ }^{3}$ George \& Fay Yee Centre for Healthcare Innovation, Winnipeg, MB, Canada. ${ }^{4}$ Information Specialist Consultant, Ottawa, Canada. ${ }^{5}$ Community Health Sciences, University of Manitoba, Winnipeg, Canada. ${ }^{6}$ Department of Haematology and Medical Oncology, CancerCare Manitoba, Winnipeg, Canada. ${ }^{7}$ Department of Internal Medicine, University of Manitoba, Winnipeg, Canada.
\end{abstract}

Received: 7 March 2017 Accepted: 7 March 2017

Published online: 17 March 2017

\section{Reference}

1. Chauhan, et al. Behavior change interventions and policies influencing primary healthcare professionals' practice-an overview of reviews. Implement Sci. 2017;12:3. doi:10.1186/s13012-016-0538-8.

\footnotetext{
*Correspondence: bchauhan28@gmail.com

${ }^{1}$ College of Pharmacy, University of Manitoba, Winnipeg, Canada

${ }^{2}$ Children's Hospital Research Institute of Manitoba, Winnipeg, Canada

Full list of author information is available at the end of the article
} 